\title{
Generation of 4-nJ Pulses from a Diode-Pumped Femtosecond $\mathrm{Ti}^{3+}$ :sapphire Laser
}

\author{
Abdullah Muti' ${ }^{1}$, Askin Kocabas ${ }^{2}$, and Alphan Sennaroglu ${ }^{1,3 *}$ \\ 1. Laser Research Laboratory, Departments of Physics and Electrical-Electronics Engineering, Koç University, Istanbul 34450, Turkey \\ 2. Department of Physics, Koç University, Istanbul 34450, Turkey \\ 3. Koç University Surface Science and Technology Center (KUYTAM), Rumelifeneri, Istanbul 34450, Turkey \\ *Corresponding author: asennar@ku.edu.tr
}

\begin{abstract}
We generated 106-fs, 4.1-nJ pulses at $778 \mathrm{~nm}$ from a single green diode-pumped multipass-cavity Kerr-lens mode-locked $\mathrm{Ti}^{3+}$ :sapphire laser. To our knowledge, these represent the highest pulse energies generated directly with a diode-pumped $\mathrm{Ti}^{3+}$ :sapphire laser.

OCIS codes: (140.4050) Mode-locked lasers; (140.7090) Ultrafast lasers; (140.3480) Lasers, diode-pumped; (140.3580)

Lasers, solid-state.
\end{abstract}

\section{Introduction}

In recent years, there has been a great deal of interest aimed at the development of cost-effective, diode-pumped 800 -nm solid-state lasers which find numerous applications in many areas of science and technology, such as biomedical imaging [1-4], spectroscopy [5], and material processing [6]. One approach has utilized AlGaInP-based red laser diodes as pump sources and gain media from the colquiriite family, which includes $\mathrm{Cr}^{3+}: \mathrm{LiSAF}$, $\mathrm{Cr}^{3+}: \mathrm{LiCAF}$, and $\mathrm{Cr}^{3+}: \operatorname{LiSGaF}[7,8]$. By using red diode pumps with different architectures, broadly tunable continuous-wave (cw) and mode-locked operation of these colquiriite lasers have been successfully demonstrated with high slope efficiencies and low lasing thresholds [7, 8]. More recently, development of high-power laser diodes in the blue and green spectral regions has also enabled the direct diode pumping of $\mathrm{Ti}^{3+}$ :sapphire lasers $[4,9-14]$. When compared with colquiriite gain media, the $\mathrm{Ti}^{3+}$ :sapphire gain medium offers additional advantages of broader tunability, negligible excited-state absorption, and reduced thermal effects [7, 15]. Direct diode pumping of $\mathrm{Ti}^{3+}$ :sapphire lasers was first demonstrated by using GaN-based pump diodes operating near $452 \mathrm{~nm}$ [9]. To date, $\mathrm{cw}$ and mode-locked operations of diode-pumped $\mathrm{Ti}^{3+}$ :sapphire lasers have been demonstrated by using blue and green pump diodes. In particular, recent studies have shown that, green AlInGaN-based laser diodes can be used to excite $\mathrm{Ti}^{3+}$ :sapphire lasers with higher efficiency near the conventional pumping wavelength of $532 \mathrm{~nm}[13,14]$. In the mode locking experiments reported by Gurel et al., as high as $1 \mathrm{~nJ}$ of pulse energy was obtained by using two, 1-W pump diodes at $520 \mathrm{~nm}[14]$.

In this study, we describe the operation of an extended-cavity, mode-locked $\mathrm{Ti}^{3+}$ :sapphire laser, directly diodepumped at $520 \mathrm{~nm}$ with one AlInGaN diode. With $780 \mathrm{~mW}$ of incident pump power, we obtained as high as $81 \mathrm{~mW}$ of cw output power from the short cavity. After adding a q-preserving multi-pass cavity, we further achieved Kerrlens mode locking and produced 106-fs pulses at a repetition rate of $5.8 \mathrm{MHz}$. The resulting energy and peak power of the pulses were $4.1 \mathrm{~nJ}$ and $39 \mathrm{~kW}$, respectively. To our knowledge, this represents the highest pulse energy directly obtained to date from a mode-locked single diode-pumped $\mathrm{Ti}^{3+}$ :sapphire laser.

\section{Experimental Setup and Results}

In the experiments, we used a Brewster-cut, 2.3-mm-long $\mathrm{Ti}^{3+}$ :sapphire crystal with a figure of merit of 51 as the gain medium. The crystal was placed inside a copper holder and kept at $22^{\circ} \mathrm{C}$ by water cooling. Initially, a standard $\mathrm{X}$-cavity was built and end pumped with a single, 1-W, 520-nm diode laser (Nichia NDG7K75T). A schematic of the experimental setup is shown in Fig. 1. The output beam of the green diode laser had different beam parameters for slow and fast axes. In particular, the divergence angle of the fast axis was 4 times larger than that for the slow axis. In order to couple the pump beam into the crystal, an aspheric collimating lens $\left(\mathrm{L}_{1}, \mathrm{f}=4 \mathrm{~mm}\right)$, a telescope with a magnification of $4\left(\mathrm{~L}_{2}\right.$ with $\mathrm{f}=25 \mathrm{~mm}$ and $\mathrm{L}_{3}$ with $\left.\mathrm{f}=100 \mathrm{~mm}\right)$ and an input focusing lens $\left(\mathrm{L}_{4}, \mathrm{f}=50 \mathrm{~mm}\right)$ were used. Here, $\mathrm{L}_{2}$ and $\mathrm{L}_{3}$ were cylindrical lenses, providing magnification only for the slow axis. To determine the optimum pumping geometry, the position of $\mathrm{L}_{1}$ and the separation of the telescope lenses $\left(\mathrm{L}_{2}\right.$ and $\left.\mathrm{L}_{3}\right)$ were varied in order to maximize the fluorescence intensity obtained at the position of the output coupler (OC). A half-wave plate (HWP) together with a polarizing beam splitter (PBS) were used in order to vary the input pump power while keeping the diode current constant at 1.6 A. The short $\mathrm{x}$-cavity consisted of a pair of curved high reflectors $\left(\mathrm{C}_{1}\right.$ and $\mathrm{C}_{2}$, each with group delay dispersion $(\mathrm{GDD})=-50 \mathrm{fs}^{2}$ and $\mathrm{R}=50 \mathrm{~mm}$ ), a flat end high reflector (shown inside the dashed rectangle) and an output coupler (OC) with $3 \%$ transmission. 


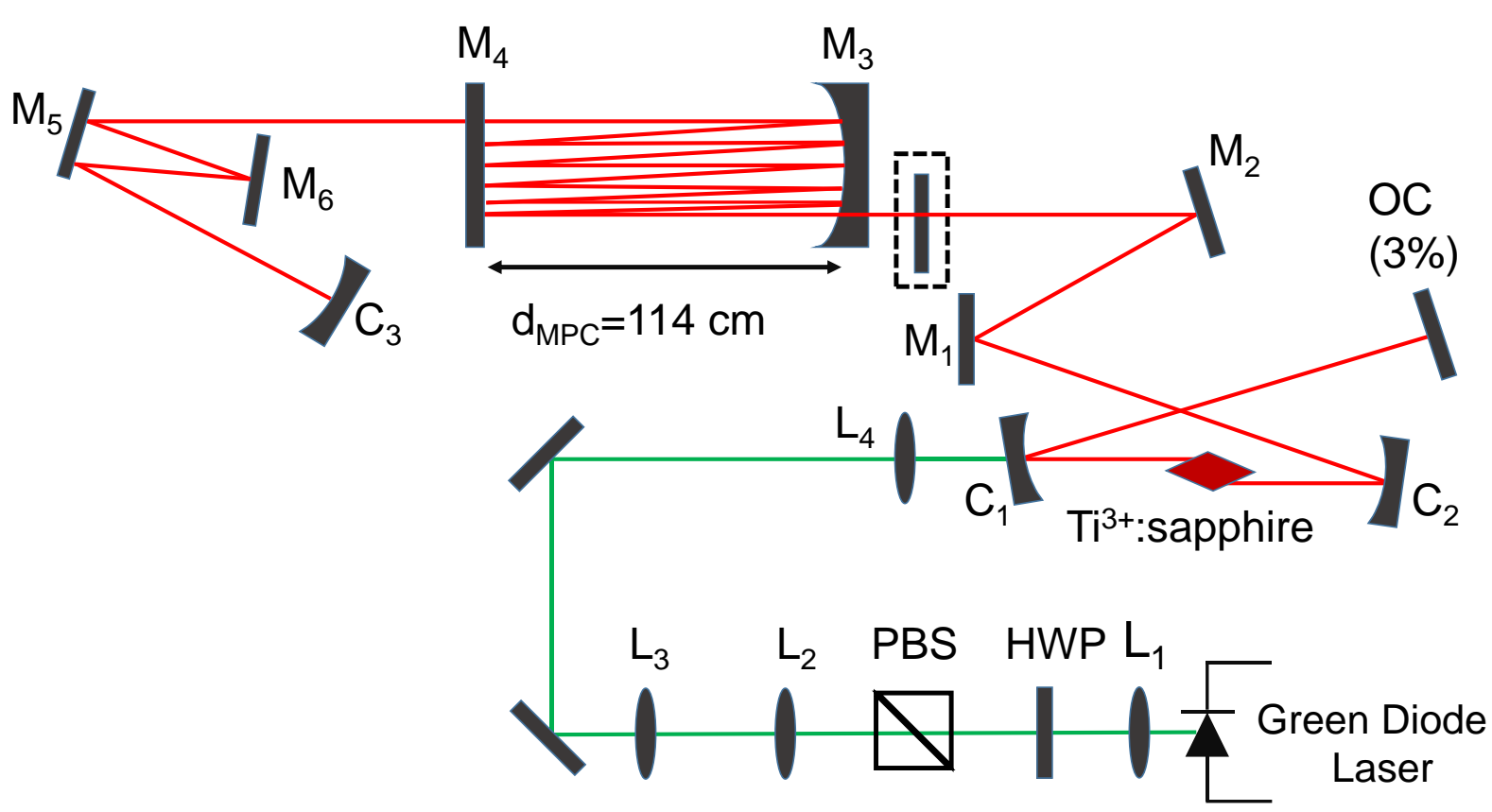

Fig. 1. Experimental setup of the single diode-pumped, multipass-cavity, femtosecond $\mathrm{Ti}^{3+}:$ sapphire laser.

After optimizing the power performance of the short x-cavity, a q-preserving multipass cavity (MPC) extension was added to reduce the pulse repetition rate [16]. The MPC consisted of a curved $\left(\mathrm{M}_{3}, \mathrm{R}=2000 \mathrm{~mm}\right)$ and a flat $\left(\mathrm{M}_{4}\right)$ high reflector, separated by $114 \mathrm{~cm} . \mathrm{M}_{3}$ and $\mathrm{M}_{4}$ further had rectangular notches for beam injection and extraction. The missing round trip due to notches was compensated by using a curved retro-reflector $\left(\mathrm{C}_{3}, \mathrm{R}=1000 \mathrm{~mm}\right)$ at a distance of $114 \mathrm{~cm}$ from $\mathrm{M}_{4}$. This q-preserving MPC configuration corresponds to $\mathrm{m}=6$ and $\mathrm{n}=11$ [16], resulting in a total round-trip optical path length of $51.7 \mathrm{~m}$ for the composite cavity. The extended cavity further contained two flat folding mirrors ( $\mathrm{M}_{1}$ with $\mathrm{GDD}=-60 \mathrm{fs}^{2}$ and $\mathrm{M}_{2}$ with $\mathrm{GDD}=-45 \mathrm{fs}^{2}$ per bounce) before $\mathrm{M}_{3}$ and two flat GTI mirrors ( $\mathrm{M}_{5}$ and $\mathrm{M}_{6}$, each with GDD=-400 fs${ }^{2}$ per bounce) between $\mathrm{M}_{4}$ and $\mathrm{C}_{3}$ for dispersion management. The total GDD of the extended cavity including the crystal and air contributions was estimated to be $-1504 \mathrm{fs}^{2}$.

Power efficiency measurements were performed for both the short (x-cavity) and the extended (MPC) resonators as shown in Fig. 2(a). During cw operation, the maximum output power was measured to be $81 \mathrm{~mW}$ and $41 \mathrm{~mW}$ for $\mathrm{x}$-cavity and MPC, respectively, at the pump power of $780 \mathrm{~mW}$. The corresponding slope efficiencies were $15 \%$ and $10 \%$.
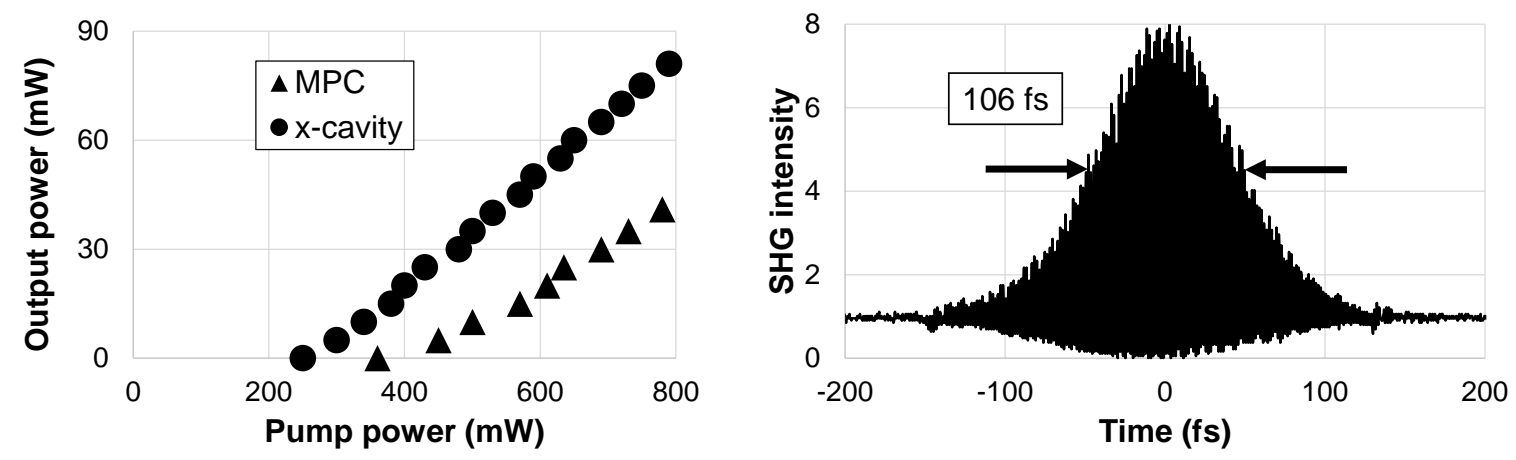

Fig. 2. (a) Continuous-wave power efficiency data for the short $\mathrm{Ti}^{3+}$ :sapphire $\mathrm{x}$-cavity and the extended resonator containing the MPC. (b) Interferometric autocorrelation of the pulses generated with the mode-locked MPC $\mathrm{Ti}^{3+}$ :sapphire laser.

In the mode locking experiments, cavity focusing around the gain crystal was optimized and the output coupler mirror was translated to generate a stable mode-locked pulse train. In order to characterize the mode-locked operation of the cavity, we measured the interferometric autocorrelation, optical spectrum, and the RF spectrum of the generated pulses as shown in Figs. 2(b) and 3. By assuming a sech ${ }^{2}$ pulse profile, the pulse duration (FWHM) was determined to be $106 \mathrm{fs}$ [Fig. 2(b)]. The spectral width (FWHM) of the pulses was $6 \mathrm{~nm}$ [Fig. 3(a)], giving a 
time-bandwidth product of 0.321 , in agreement with the assumed $\operatorname{sech}^{2}$ intensity distribution. The RF measurements indicated that the fundamental beat note at $5.8 \mathrm{MHz}$ was $63 \mathrm{dBc}$ above the noise level. At the pump power of 750 $\mathrm{mW}$, a maximum output power of $24 \mathrm{~mW}$ was obtained during mode-locked operation, corresponding to $4.1 \mathrm{~nJ}$ of pulse energy and $39 \mathrm{~kW}$ of peak power.
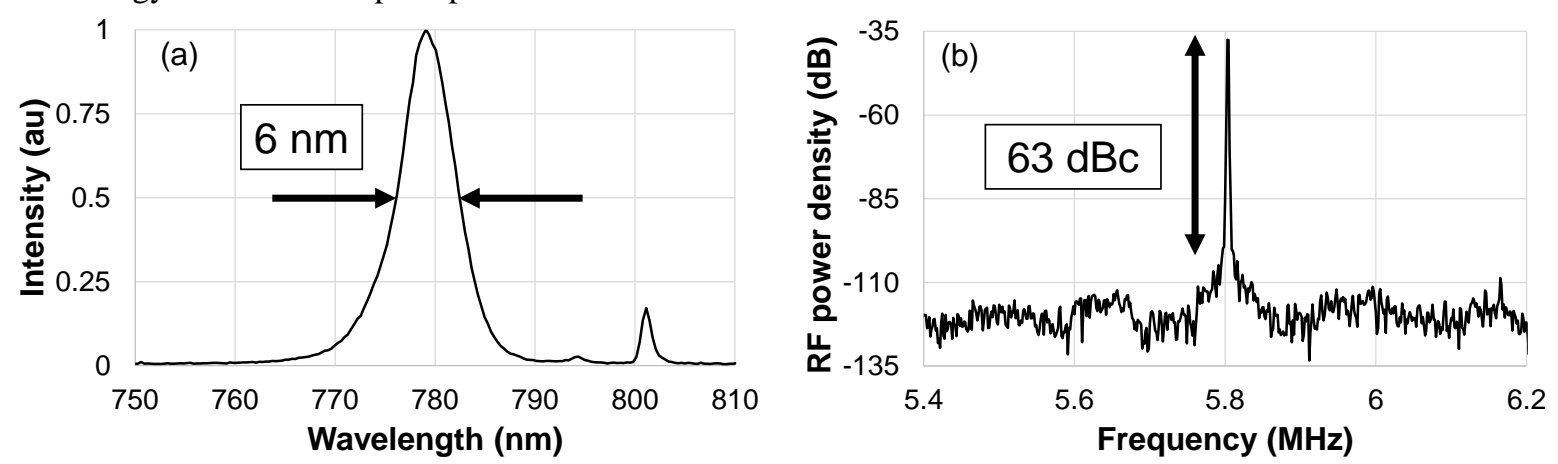

Fig. 3. (a) Mode-locked optical spectrum and (b) radio frequency (RF) spectrum of the pulses generated with the diode-pumped mode-locked MPC $\mathrm{Ti}^{3+}$ :sapphire laser. At the pulse repetition rate of $5.8 \mathrm{MHz}$, the fundamental beat note was $63 \mathrm{dBc}$ above the noise level.

In conclusion, we generated 106-fs pulses with $4.1 \mathrm{~nJ}$ of pulse energy at $778 \mathrm{~nm}$ from a single diode-pumped multipass-cavity Kerr-lens mode-locked $\mathrm{Ti}^{3+}$ :sapphire laser. To the best of our knowledge, this represents the highest pulse energy directly generated from a diode-pumped $\mathrm{Ti}^{3+}$ :sapphire laser. We believe that further energy scaling should be possible by using more pump diodes.

\section{References}

[1] W. R. Zipfel, R. M. Williams, and W. W. Webb, "Nonlinear magic: multiphoton microscopy in the biosciences," Nat. Biotechnol. 21, 1369-1377, (2003).

[2] D. Huang, E. A. Swanson, C. P. Lin, J. S. Schuman, W. G. Stinson, W. Chang, M. R. Hee, T. Flotte, K. Gregory, C. A. Puliafito, and J. G. Fujimoto, "Optical Coherence Tomography," Science 254, 1178-1181, (1991).

[3] S. Sakadžić, U. Demirbas, T. R. Mempel, A. Moore, S. Ruvinskaya, D. A. Boas, A. Sennaroglu, F. X. Kartner, and J. G. Fujimoto, "Multi-photon microscopy with a low-cost and highly efficient Cr:LiCAF laser," Opt. Express 16, 20848-20863, (2008).

[4] M. D. Young, S. Backus, C. Durfee, and J. Squier, "Multiphoton imaging with a direct-diode pumped femtosecond Ti:sapphire laser," J. Microsc.-Oxford 249, 83-86, (2013).

[5] D. W. McCamant, P. Kukura, S. Yoon, and R. A. Mathies, "Femtosecond broadband stimulated Raman spectroscopy: Apparatus and methods," Rev. Sci. Instrum. 75, 4971-4980, (2004).

[6] X. Liu, D. Du, and G. Mourou, "Laser ablation and micromachining with ultrashort laser pulses," IEEE J. Quantum Elect. 33, 17061716, (1997).

[7] U. Demirbas, D. Li, J. R. Birge, A. Sennaroglu, G. S. Petrich, L. A. Kolodziejski, F. X. Kartner, and J. G. Fujimoto, "Low-cost, single-mode diode-pumped Cr:Colquiriite lasers," Opt. Express 17, 14374-14388, (2009).

[8] U. Demirbas, G. S. Petrich, D. Li, A. Sennaroglu, L. A. Kolodziejski, F. X. Kartner, and J. G. Fujimoto, "Femtosecond tuning of Cr:colquiriite lasers with AlGaAs-based saturable Bragg reflectors," J. Opt. Soc. Am. B 28, 986-993, (2011).

[9] P. W. Roth, A. J. Maclean, D. Burns, and A. J. Kemp, "Directly diode-laser-pumped Ti:sapphire laser," Opt. Lett. 34, 3334-3336, (2009).

[10] P. W. Roth, A. J. Maclean, D. Burns, and A. J. Kemp, "Direct diode-laser pumping of a mode-locked Ti:sapphire laser," Opt. Lett. 36, 304-306, (2011).

[11] P. W. Roth, D. Burns, and A. J. Kemp, "Power scaling of a directly diode-laser-pumped Ti:sapphire laser," Opt. Express 20, 2062920634, (2012).

[12] C. G. Durfee, T. Storz, J. Garlick, S. Hill, J. A. Squier, M. Kirchner, G. Taft, K. Shea, H. Kapteyn, M. Murnane, and S. Backus, "Direct diode-pumped Kerr-lens mode-locked Ti:sapphire laser," Opt. Express 20, 13677-13683, (2012).

[13] S. Sawai, A. Hosaka, H. Kawauchi, K. Hirosawa, and F. Kannari, "Demonstration of a Ti:sapphire mode-locked laser pumped directly with a green diode laser," Appl. Phys. Express 7, (2014).

[14] K. Gurel, V. J. Wittwer, M. Hoffmann, C. J. Saraceno, S. Hakobyan, B. Resan, A. Rohrbacher, K. Weingarten, S. Schilt, and T. Sudmeyer, "Green-diode-pumped femtosecond Ti:Sapphire laser with up to $450 \mathrm{~mW}$ average power," Opt. Express 23, 30043-30048, (2015).

[15] P. F. Moulton, "Spectroscopic and Laser Characteristics of Ti-Al2O3," J. Opt. Soc. Am. B 3, 125-133, (1986).

[16] A. Sennaroglu, A. M. Kowalevicz, E. P. Ippen, and J. G. Fujimoto, "Compact femtosecond lasers based on novel multipass cavities," IEEE J. Quantum Elect. 40, 519-528, (2004). 